\title{
NONHOMOGENEOUS BOUNDARY VALUE PROBLEM FOR A SEMILINEAR HYPERBOLIC EQUATION
}

Abstract. We discuss the solvability of a nonhomogeneous boundary value problem for the semilinear equation of the vibrating string $x_{t t}(t, y)-$ $\Delta x(t, y)+f(t, y, x(t, y))=0$ in a bounded domain and with a certain type of superlinear nonlinearity. To this end we derive a new dual variational method.

1. Introduction. Throughout the paper, $\Omega$ will be a general open bounded domain in $\mathbb{R}^{n}$, with boundary $\Gamma$, assumed to be smooth. The aim of the paper is to study the existence, in a finite interval $[0, T]$, of a solution of the following second order hyperbolic semilinear problem with Dirichlet boundary condition:

$$
\begin{aligned}
& x_{t t}(t, y)-\Delta x(t, y)+f(t, y, x(t, y))=0, \\
& x(0, y)=x^{0}(y), \quad x_{t}(0, y)=x^{1}(y), \quad y \in \Omega, \\
& x(t, y)=u(t, y), \quad(t, y) \in \Sigma=(0, T) \times \Gamma .
\end{aligned}
$$

It is worth noting here that the nonlinearity $f$, generally, may drive the solution of (1) to blow up in finite time [4], [10], [5], [1] (see also discussion in [2] and [11]). We exclude such a case assuming hypothesis G4, which imposes some bound on the growth of $f$ at time $T$. The importance of the problem with control on the boundary can be seen in optimal control theory (see e.g. [8]).

We shall study (1) by the classical variational method, i.e. we shall consider (1) as the Euler-Lagrange equation of the action functional

2000 Mathematics Subject Classification: Primary 35L05, 35L20; Secondary 35A15.

Key words and phrases: nonhomogeneous boundary value problem, semilinear equation of vibrating string, dual variational method. 


$$
\begin{aligned}
J(x)= & \int_{0}^{T} \int_{\Omega}\left(\frac{1}{2}|\nabla x(t, y)|^{2}-\frac{1}{2}\left|x_{t}(t, y)\right|^{2}+F(t, y, x(t, y))\right) d y d t \\
& -\int_{\Omega} x(T, y) x^{1}(y) d y,
\end{aligned}
$$

where $F_{x}=f$, defined on some subspace of $C\left([0, T] ; H^{2}(\Omega)\right)$ discussed below. The last integral in (2) relates to the fact that we consider initial conditions instead of, as is usual in the calculus of variations, the two-point boundary problem. In many papers (see e.g. [11] and the references therein) another functional is considered, the so called energy functional, associated with (1), to get some estimates for solutions of (1).

Our purpose is to investigate (1) by studying the critical points of the functional (2). To this end we apply a new duality approach which is based on ideas developed in [9]; however, we drop some unpleasant assumptions appearing in [9], e.g. convexity of $F(t, y, \cdot)$. As mentioned above, the functional (2) is unbounded in $C\left([0, T] ; H^{2}(\Omega)\right)$ and this is why we are looking for critical points of $J$ of min-max type. Our aim is to find a subset $X$ of $C\left([0, T] ; H^{2}(\Omega)\right)$ and study (2) only on $X$. The main difficulty in our approach is the construction of $X$. The one-dimensional case of (1) under the convexity assumption of $F(t, y, \cdot)$ was described in [9].

The plan of the paper is as follows: in the Preliminaries we define all objects we need later, give hypotheses we use and recall the linear case from [6]; in the next section we prove auxiliary results; then the duality theory is developed for our problem and necessary conditions for a minimizer of $J$ in $X$ to exist are proved. In the last section we prove the existence of a minimizer of $J$ in $X$.

2. Preliminaries. Let $\mathcal{L}=\left\{g \in L^{1}\left(0, T ; H^{1}(\Omega)\right): g_{t} \in L^{1}\left(0, T ; H^{0}(\Omega)\right)\right\}$, $x^{0} \in H^{2}(\Omega), x^{1} \in H^{1}(\Omega)$ and let

$$
U^{1}=C\left([0, T] ; H^{2}(\Omega)\right), \quad U^{2}=C\left([0, T] ; H^{1}(\Omega)\right)
$$

and

$$
\begin{gathered}
U=\left\{x \in C\left([0, T] ; H^{2}(\Omega)\right): \frac{\partial x}{\partial t} \in C\left([0, T] ; H^{1}(\Omega)\right), \frac{\partial^{2} x}{\partial t^{2}} \in C\left([0, T] ; H^{0}(\Omega)\right),\right. \\
\left.x(0, \cdot)=x^{0}(\cdot), x_{t}(0, \cdot)=x^{1}(\cdot), x(t, y)=u(t, y),(t, y) \in \Sigma=(0, T) \times \Gamma\right\} .
\end{gathered}
$$

Observe that each $x \in U$ has the derivatives $\partial^{2} x / \partial t^{2}, \partial^{2} x / \partial y_{i}^{2}, i=1, \ldots, n$, for almost every $(t, y) \in[0, T] \times \Omega$. Since we are looking for solutions of (1) in some subset of $U$, by a solution of (1) we mean $x \in U$ which satisfies (1) almost everywhere. We shall consider $\mathcal{L}$ with the norm

$$
|g|_{\mathcal{L}}^{2}=|g|_{L^{1}\left(0, T ; H^{1}(\Omega)\right)}^{2}+\left|g_{t}\right|_{L^{1}\left(0, T ; H^{0}(\Omega)\right)}^{2},
$$


$U$ with the norm

$$
|x|_{U}^{2}=|x|_{C\left([0, T] ; H^{2}(\Omega)\right)}^{2}+\left|x_{t}\right|_{C\left([0, T] ; H^{1}(\Omega)\right)}^{2}+\left|x_{t t}\right|_{C\left([0, T] ; H^{0}(\Omega)\right)}^{2}
$$

and $U^{1}, U^{2}$ with the norms

$$
|x|_{U^{1}}^{2}=|x|_{C\left([0, T] ; H^{2}(\Omega)\right)}^{2}, \quad|x|_{U^{2}}^{2}=|x|_{C\left([0, T] ; H^{1}(\Omega)\right)}^{2} .
$$

In our notation Theorem 2.2 from [6] has the following form:

Theorem 1. Let $g \in \mathcal{L}, u \in H^{2}(\Sigma), x^{0} \in H^{2}, x^{1} \in H^{1}$. Then there exists a unique solution $\bar{x} \in U$ to

$$
\begin{aligned}
& x_{t t}(t, y)-\Delta x(t, y)=g(t, y), \\
& x(0, y)=x^{0}(y), \quad x_{t}(0, y)=x^{1}(y), y \in \Omega, \\
& x(t, y)=u(t, y), \quad(t, y) \in \Sigma,
\end{aligned}
$$

such that

$$
\begin{aligned}
|\bar{x}|_{U^{1}} & \leq B\left(|g|_{\mathcal{L}}+|u|_{H^{2}(\Sigma)}+\left|x^{0}\right|_{H^{2}(\Omega)}+\left|x^{1}\right|_{H^{1}(\Omega)}\right), \\
\left|\bar{x}_{t}\right|_{U^{2}} & \leq D\left(|g|_{\mathcal{L}}+|u|_{H^{2}(\Sigma)}+\left|x^{0}\right|_{H^{2}(\Omega)}+\left|x^{1}\right|_{H^{1}(\Omega)}\right)
\end{aligned}
$$

with some $B, D>0$ independent of $g$ and with the compatibility conditions $\left.u\right|_{t=0}=\left.x^{0}\right|_{\Gamma},\left.\frac{\partial u}{\partial t}\right|_{t=0}=\left.x^{1}\right|_{\Gamma}$.

The fact that $x \in C\left([0, T] ; H^{2}(\Omega)\right)$ is explained in [6, pp. 153-154].

The solution $\bar{x}$ from the theorem may also be estimated by

$$
\begin{aligned}
|\bar{x}(t, \cdot)|_{H^{1}(\Omega)} & \leq|\bar{x}(t, \cdot)|_{H^{2}(\Omega)} \leq T B \sup _{\tau \in(0, T)}|g(\tau, \cdot)|_{H^{1}(\Omega)}+E, \\
\left|\bar{x}_{t}(t, \cdot)\right|_{H^{1}(\Omega)} & \leq T D \sup _{\tau \in(0, T)}|g(\tau, \cdot)|_{H^{1}(\Omega)}+A
\end{aligned}
$$

where $E=B\left(|u|_{H^{2}(\Sigma)}+\left|x^{0}\right|_{H^{2}(\Omega)}+\left|x^{1}\right|_{H^{1}(\Omega)}\right)$ and $A=D\left(|u|_{H^{2}(\Sigma)}+\right.$ $\left.\left|x^{0}\right|_{H^{2}(\Omega)}+\left|x^{1}\right|_{H^{1}(\Omega)}\right)$ and we will just use these last estimates.

We assume the following hypotheses:

G1. There exists a function $\bar{z} \in C\left([0, T] ; H^{2}(\Omega)\right)$ such that $F_{x}(\bar{z}) \in \mathcal{L}$ $\left(F_{x}(h)=F_{x}(\cdot, \cdot, h(\cdot, \cdot))\right)$; put

$$
I=\left[-E-T B \sup _{\tau \in(0, T)}|\bar{z}(\tau, \cdot)|_{H^{2}(\Omega)}, T B \sup _{\tau \in(0, T)}|\bar{z}(\tau, \cdot)|_{H^{2}(\Omega)}+E\right],
$$

G2. $F$ is differentiable with respect to the third variable.

G3. $F_{x}(t, y, 0) \neq 0$ for a.e. $(t, y) \in(0, T) \times \Omega ;(t, y) \mapsto F(t, y, 0)$ is integrable on $(0, T) \times \Omega ; F_{x}(t, y, \cdot)$ is continuous in $\mathbb{R}$ for a.e. $(t, y) \in$ $(0, T) \times \Omega$.

G4. For $x \in U$ such that $|x(t, \cdot)|_{H^{2}(\Omega)} \in I$ for all $t \in(0, T)$, we have $F_{x}(\cdot, \cdot, x(\cdot, \cdot)) \in \mathcal{L}, F_{x}(\cdot, \cdot, x(\cdot, \cdot)) \in L^{2}(\Sigma)$, and 


$$
\begin{aligned}
\left|F_{x}(t, \cdot, x(t, \cdot))\right|_{H^{1}(\Omega)} & \leq \sup _{\tau \in(0, T)}|\bar{z}(\tau, \cdot)|_{H^{2}(\Omega)}, t \in(0, T), \\
F(t, y, x) & \geq a(t, y) x+b(t, y),
\end{aligned}
$$

for some $a, b \in L^{2}((0, T) \times \Omega)$ and all $x \in \mathbb{R}$.

CC. $u \in H^{2}(\Sigma)$ and $\left.u\right|_{t=0}=\left.x^{0}(\cdot)\right|_{\Gamma},\left.\quad \frac{\partial u}{\partial t}\right|_{t=0}=\left.x^{1}(\cdot)\right|_{\Gamma}$.

It is clear that the form of $F_{x}$ depends (locally) strongly on the function $\bar{z}$. Moreover, G4 guarantees that the functional (2) is bounded in the set $\bar{X}$ defined below (see Lemma 2) and that solutions to (1) (if they exist) are bounded (see Lemma 1). An example of $F_{x}$ satisfying $\mathbf{G 1 - G 4}$ is given below.

EXAmple. Choose $F_{x}(t, y, x)=a(t)\left(x^{5}-(1 / 2) x^{4}\right), x^{0}$ and $x^{1}$ such that $\left|x^{0}\right|_{H^{2}(\Omega)}=1 / 8$ and $\left|x^{1}\right|_{H^{1}(\Omega)}=1 / 8, u=0, \Omega=(0, \pi), T=1,0<$ $a(t)<1 / 3, a(\cdot) \in C^{3}(0, T), \bar{z}$ such that $\sup _{t \in(0, T)}|\bar{z}(t, \cdot)|_{H^{2}(\Omega)}=0.7$ and $B=1$. Then $E=1 / 4$ and $I=[-0.95,0.95]$, so $|x(t, \cdot)|_{H^{2}(\Omega)} \in I$ means that $|x(t, \cdot)|_{H^{2}(\Omega)}<1$ and thus $|x(t, \cdot)|_{H^{1}(\Omega)}<1,|x(t, \cdot)|_{L^{2}(\Omega)}<1$. Hence, for those $x(\cdot, \cdot)$,

$$
\begin{aligned}
|f(t, \cdot, x(t, \cdot))|_{H^{1}(\Omega)} & \leq|a(t)||x(t, \cdot)|_{H^{1}(\Omega)}^{4}|x(t, \cdot)-1 / 2|_{H^{1}(\Omega)}<|a(t)| 2<0.7 \\
& =\sup _{t \in(0, T)}|\bar{z}(t, \cdot)|_{H^{2}(\Omega)} .
\end{aligned}
$$

Moreover,

$$
a(t) x^{5}((1 / 6) x-1 / 10) \geq-1, \quad x \in \mathbb{R}, t \in[0,1], y \in(0, \pi) .
$$

Since, for each $t \in[0,1], y \in(0, \pi), x \mapsto a(t) x^{5}((1 / 6) x-(1 / 10))$ is convex in $\mathbb{R}$, the functional

$$
x \mapsto \int_{0}^{T} \int_{\Omega} a(t) x^{5}((1 / 6) x-1 / 10) d y d t
$$

is convex in the space $L^{2}\left(0, T ; L^{2}(\Omega)\right)$ and has nonempty subdifferential for $x \in L^{2}\left(0, T ; L^{2}(\Omega)\right)$ (see the assumptions of Theorem 2).

Define $\bar{X}=\left\{x \in U:|x(t, \cdot)|_{H^{2}(\Omega)} \in I, t \in(0, T)\right\}$. We shall consider the functional $J$ of (2) on

$$
\begin{aligned}
\widetilde{U}=\left\{x \in C\left([0, T] ; H^{2}(\Omega)\right): \partial x / \partial t \in C\left([0, T] ; H^{1}(\Omega)\right),\right. \\
\left.x(0, \cdot)=x^{0}(\cdot), x_{t}(0, \cdot)=x^{1}(\cdot)\right\} .
\end{aligned}
$$

Note that $J$ is bounded on $\bar{X}$ (see also Lemma 2), thus we need to look for a kind of "critical point" in $\bar{X}$. To this end we shall consider the dual functional, which will be investigated together with (2): 


$$
\begin{aligned}
J_{D}(p, q)= & -\int_{0}^{T} \int_{\Omega} F^{*}\left(t, y, p_{t}(T-t, y)+\operatorname{div} q(t, y)\right) d y d t \\
& -\frac{1}{2} \int_{0}^{T} \int_{\Omega}|q(t, y)|^{2} d y d t+\frac{1}{2} \int_{0}^{T} \int_{\Omega}|p(T-t, y)|^{2} d y d t \\
& +\int_{\Omega} x^{0}(y) p(T, y) d y d t-\int_{\Sigma} u(t, y)\langle q(t, y), \nu(y)\rangle d y d t
\end{aligned}
$$

where $\nu=\left(\nu_{1}, \ldots, \nu_{n}\right)$ is the unit outward normal to $\Gamma, F^{*}$ is the FenchelYoung conjugate to $F\left(h^{*}\left(s^{*}\right)=\sup _{s}\left\{\left\langle s^{*}, s\right\rangle-h(s)\right\}\right.$; see e.g. [3]) and

$$
\begin{array}{r}
J_{D}: U_{D}=\left\{(p, q): p \in C\left([0, T] ; H^{1}(\Omega)\right), \partial p / \partial t \in C\left([0, T] ; H^{0}(\Omega)\right),\right. \\
\left.p(0, \cdot)=x^{1}(\cdot), q \in L^{2}\left(0, T ; H_{n}^{1}(\Omega)\right)\right\} \rightarrow \mathbb{R} .
\end{array}
$$

We will consider two kinds of relationship between the functionals $J$ and $J_{D}$ on $X$ : the Duality Principle and the Variational Principle. The former, Theorem 3, relates the critical values of both functionals, while the latter, Theorem 4, provides necessary conditions that must be satisfied by the solution to problem (1).

3. The auxiliary results. Now we construct the sets on which $J$ and $J_{D}$ will be considered. In view of the definition of $\bar{X}$ and Theorem 1 the following lemma can be formulated:

Lemma 1. There exist constants $C_{1}, C_{2}, C_{3}, C_{4}$ independent of $x \in \bar{X}$ such that

$$
\begin{aligned}
|v|_{L^{\infty}\left(0, T ; H^{2}(\Omega)\right)} & \leq C_{2}, \quad\left|v_{t}\right|_{L^{\infty}\left(0, T ; H^{1}(\Omega)\right)} \leq C_{1}, \\
\left|v_{t t}\right|_{L^{\infty}\left(0, T ; L^{2}(\Omega)\right)} & \leq C_{3}, \quad|\Delta v|_{L^{\infty}\left(0, T ; L^{2}(\Omega)\right)} \leq C_{2}, \\
|v|_{H^{2}((0, T) \times \Omega)} & \leq C_{4},
\end{aligned}
$$

where $v$ is the solution of the problem

$$
\begin{aligned}
& v_{t t}(t, y)-\Delta v(t, y)=-F_{x}(t, y, x(t, y)) \quad \text { a.e. on }(0, T) \times \Omega, \\
& v(0, y)=x^{0}(y), \quad v_{t}(0, y)=x^{1}(y), \quad y \in \Omega, \\
& v(t, y)=u(t, y), \quad(t, y) \in \Sigma,
\end{aligned}
$$

with $x \in \bar{X}$.

Proof. Fix $x \in \bar{X}$. Since $x \in U$ and by the assumptions on $F$ (see G4), it follows that $F_{x}(\cdot, \cdot, x(\cdot, \cdot)) \in \mathcal{L}$. Hence by Theorem 1 and (6) there exists a unique solution $v \in U$ of problem (10) satisfying

$$
|v|_{U^{1}} \leq T B\left|F_{x}(x)\right|_{L^{\infty}\left(0, T ; H^{1}(\Omega)\right)}+E .
$$


Taking into account $\mathbf{G} 4$ we get the following estimate for some $C$, independent of $x \in \bar{X}$ :

$$
T\left|F_{x}(x)\right|_{L^{\infty}\left(0, T ; H^{1}(\Omega)\right)} \leq C .
$$

Moreover, by (6) we get

$$
|v|_{L^{\infty}\left(0, T ; H^{2}(\Omega)\right)} \leq T B\left|F_{x}(x)\right|_{L^{\infty}\left(0, T ; H^{1}(\Omega)\right)}+E \leq B C+E .
$$

By (7),

$$
\left|v_{t}\right|_{L^{\infty}\left(0, T ; H^{1}(\Omega)\right)} \leq T D\left|F_{x}(x)\right|_{L^{\infty}\left(0, T ; H^{1}(\Omega)\right)}+A \leq D C+A .
$$

Since $|\Delta v|_{H^{0}(\Omega)} \leq|v|_{H^{2}(\Omega)}$, we have $\left|v_{t t}\right|_{L^{\infty}\left(0, T ; H^{0}(\Omega)\right)} \leq(B C+E)+C / T$. Hence, putting

$$
\begin{aligned}
& C_{2}=B C+E, \quad C_{1}=D C+A, \quad C_{3}=(B C+E)+C / T, \\
& C_{4}=T^{1 / 2}(3 B C+E)+(B C+E)+C / T+(D C+A),
\end{aligned}
$$

we obtain the assertion of the lemma.

Proposition 1. For every $x \in \bar{X}$ the relations

$$
\begin{aligned}
& \widetilde{x}_{t t}(t, y)-\Delta \widetilde{x}(t, y)=-F_{x}(t, y, x(t, y)), \\
& \widetilde{x}(0, y)=x^{0}(y), \quad \widetilde{x}_{t}(0, y)=x^{1}(y), \quad y \in \Omega, \\
& \widetilde{x}(t, y)=u(t, y), \quad(t, y) \in \Sigma,
\end{aligned}
$$

imply that $\widetilde{x} \in \bar{X}$.

Proof. Fix $x \in X$. Since $x \in U$ and by the assumptions on $F$ (see G4), it follows that $F_{x}(\cdot, \cdot, x(\cdot, \cdot)) \in \mathcal{L}$. Hence by Theorem 1 there exists a unique solution $\widetilde{x} \in U$ of problem (11). Moreover, $\widetilde{x}_{t t}-\Delta \widetilde{x} \in \mathcal{L}$. Indeed, by G4, it follows that

$$
\left|F_{x}(t, \cdot, x(t, \cdot))\right|_{H^{1}(\Omega)} \leq \sup _{t \in(0, T)}|\bar{z}(t, \cdot)|_{H^{2}(\Omega)} \cdot
$$

Further, by (6) we get $|\widetilde{x}|_{U^{1}} \leq T B \sup _{t \in(0, T)}\left|F_{x}(t, \cdot, x(t, \cdot))\right|_{H^{1}(\Omega)}+E$. Now by (12) it follows that

$$
|\widetilde{x}|_{U^{1}} \leq T B \sup _{t \in(0, T)}|\bar{z}(t, \cdot)|_{H^{2}(\Omega)}+E .
$$

Hence

$$
|\widetilde{x}(t, \cdot)|_{H^{2}(\Omega)} \leq T B \sup _{t \in(0, T)}|\bar{z}(t, \cdot)|_{H^{2}(\Omega)}+E, \quad t \in(0, T) .
$$

So $|\widetilde{x}(t, \cdot)|_{H^{2}(\Omega)} \in I, t \in(0, T)$. Thus for every $x \in \bar{X}$ there exists an $\widetilde{x} \in \bar{X}$ satisfying (11).

Let $H$ be the map assigning to $x \in \bar{X}$ the solution $\widetilde{x} \in \bar{X}$ of (11). Define $X=H(\bar{X})$.

Corollary 1. Every $\left\{x_{n}\right\} \subset X$ has a subsequence converging weakly in $L^{2}\left(0, T ; H^{2}(\Omega)\right)$ and $H^{2}((0, T) \times \Omega)$ and strongly in $L^{2}\left(0, T ; H^{1}(\Omega)\right)$. 
Moreover, there is a subsequence $\left\{x_{n_{j}}\right\}$ of $\left\{x_{n}\right\}$ such that $\left\{x_{n_{j} t}\right\},\left\{x_{n_{j} y_{i}}\right\}$, $i=1, \ldots, n,\left(y=\left(y_{1}, \ldots, y_{n}\right)\right)$ are strongly convergent in $L^{2}((0, T) \times \Omega)$.

Proof. Let $\left\{x_{n}\right\} \subset X$. Then by the above proposition each $x_{n}$ is a solution of problem (11). Hence by Lemma 1 the sequence $\left\{x_{n}\right\}$ is bounded in $H^{2}((0, T) \times \Omega)$, in $L^{\infty}\left(0, T ; H^{2}(\Omega)\right)$ and so in $L^{2}\left(0, T ; H^{2}(\Omega)\right),\left\{x_{n t}\right\}$ in $L^{\infty}\left(0, T ; H^{1}(\Omega)\right)$ and $\left\{x_{n t t}\right\}$ in $L^{\infty}\left(0, T ; H^{0}(\Omega)\right)$. Therefore $\left\{x_{n}\right\}$ has a weakly convergent subsequence in $L^{2}\left(0, T ; H^{2}(\Omega)\right)$ and in $H^{2}((0, T) \times \Omega)$. Since all $x_{n t}$ are bounded by the same constant, the above-mentioned subsequence converges strongly in $L^{2}\left(0, T ; H^{1}(\Omega)\right)$. Moreover $x_{n t t}, x_{n y_{i} y_{j}}, x_{n t y_{j}}$, $i, j=1, \ldots, n$, are also bounded in $L^{2}((0, T) \times \Omega)$ by the same constant. Hence there is a subsequence $\left\{x_{n_{j}}\right\}$ of $\left\{x_{n}\right\}$ such that $\left\{x_{n_{j} t}\right\},\left\{x_{n_{j} y_{i}}\right\}, i=$ $1, \ldots, n$, are strongly convergent in $L^{2}((0, T) \times \Omega)$.

Now we define the set on which the dual action functional will be considered. Define

$$
\begin{aligned}
W_{t}^{1} & =W_{t}^{1}((0, T) \times \Omega) \\
& =\left\{p \in C\left([0, T] ; H^{1}(\Omega)\right): p_{t} \in C\left([0, T] ; H^{0}(\Omega)\right), p(0, \cdot)=x^{1}(\cdot)\right\}
\end{aligned}
$$

and

$$
\begin{aligned}
W_{y}^{1} & =W_{y}^{1}((0, T) \times \Omega) \\
& =\left\{q \in L^{2}\left(0, T ; H_{n}^{1}(\Omega)\right): \operatorname{div} q \in L^{2}\left(0, T ; H^{0}(\Omega)\right)\right\} .
\end{aligned}
$$

Definition 1. We say that an element $(p, q) \in W_{t}^{1} \times W_{y}^{1}$ belongs to $X^{d}$ provided that there exists $x \in X$ such that for a.e. $(t, y) \in(0, T) \times \Omega$,

$$
-p_{t}(T-t, y)-\operatorname{div} q(t, y)=-F_{x}(t, y, x(t, y)) \text { with } q(t, y)=\nabla x(t, y)
$$

or else

$$
p(T-t, y)=x_{t}(t, y) \quad \text { with } q(t, y)=\nabla x(t, y) .
$$

Remark 1. The definition of $X^{d}$ says that for each $x \in X$ there exist in $X^{d}$ two pairs of $(p, q)$ : one defined by (14), the other by (15).

We will use the sets

$$
X_{1}^{d}=\left\{p \in W_{t}^{1}:(p, q) \in X^{d}\right\}, \quad X_{2}^{d}=\left\{q \in W_{y}^{1}:(p, q) \in X^{d}\right\} .
$$

We observe that neither $X$ nor $X^{d}$ is a linear space. Thus even standard calculations using convexity arguments - the tool which is indispensable if one wants to apply the variational approach - are rather difficult. What helps us is a special structure of the sets $X$ and $X^{d}$ which despite their nonlinearity makes these calculations possible.

Notice that the functionals $J$ and $J_{D}$ are well defined on $X$ and $X^{d}$. 
Lemma 2. There exist constants $M_{1}, M_{2}$ such that, for all $x \in X$,

$$
M_{1} \leq \int_{0}^{T} \int_{\Omega} F(t, y, x(t, y)) d y d t \leq M_{2} .
$$

Proof. The first inequality in (16) is a direct consequence of G4. By the mean value theorem and $\mathbf{G} 4$ we have, for some $0<\theta<1$,

$$
\begin{aligned}
F(t, y, & x(t, y)) \leq F(t, y, 0)+|x(t, y)|\left|F_{x}(t, y, \theta x(t, y))\right| \\
& \leq F(t, y, 0)+|x(t, \cdot)|_{H^{1}(\Omega)} \sup _{v \in X}\left|F_{x}(t, \cdot, v(t, \cdot))\right|_{H^{1}(\Omega)} \\
& \leq F(t, y, 0)+\sup _{t \in(0, T)}|\bar{z}(t, \cdot)|_{H^{2}(\Omega)}\left\{T B \sup _{t \in(0, T)}|\bar{z}(t, \cdot)|_{H^{2}(\Omega)}+E\right\} .
\end{aligned}
$$

Hence we infer the boundedness from above in (16).

Now we may state the main result of the paper which is the following existence theorem.

Theorem 2. There exists $\bar{x} \in X$ such that $\inf _{x \in X} J(x)=J(\bar{x})$. Assume that the functional $x \mapsto \int_{0}^{T} \int_{\Omega} F(t, y, x(t, y)) d y d t$ is subdifferentiable at the point $\bar{x}$ (the subdifferential in the sense of convex analysis is taken in the space $\left.L^{2}\left(0, T ; L^{2}(\Omega)\right)\right)$. Then there exist $(\bar{p}, \bar{q}) \in X^{d}$ such that

$$
J_{D}(\bar{p}, \bar{q})=\inf _{x \in X} J(x)=J(\bar{x})
$$

and

$$
\begin{aligned}
\bar{x}_{t}(t, y) & =\bar{p}(T-t, y), \\
\nabla \bar{x}(t, y) & =\bar{q}(t, y), \\
-\bar{p}_{t}(T-t, y)-\operatorname{div} \bar{q}(t, y) & =-F_{x}(t, y, \bar{x}(t, y)) .
\end{aligned}
$$

The system (18)-(20) may be viewed as a system of Hamiltonian equations and its existence will be obtained with the aid of a duality theory.

REMARK 2. The system (18)-(20) is equivalent to (1). Indeed, as $\bar{x} \in X$ we have $\bar{x} \in U$. Hence $\bar{x}$ satisfies the initial and boundary conditions. Of course, by $(18), \bar{x}_{t t}(t, y)=-\bar{p}_{t}(T-t, y)$, and by $(19), \operatorname{div} \nabla \bar{x}(t, y)=$ $\operatorname{div} \bar{q}(t, y)$. Putting both equalities in (20) we get (1). The converse implication is obvious.

4. Duality result. We shall construct a duality theory for the functional $J_{D}: X^{d} \rightarrow \mathbb{R}$. To avoid the calculation of the Fenchel-Young transform with respect to a subset we introduce a perturbation functional defined on the 
whole space. Let $J_{p}: X^{d} \times L^{2}\left((0, T) \times \Omega ; \mathbb{R}^{n}\right) \rightarrow \mathbb{R}$ be given by the formula

$$
\begin{aligned}
J_{p}(p, q, v)= & \int_{0}^{T} \int_{\Omega} F^{*}\left(t, y, p_{t}(T-t, y)+\operatorname{div} q(t, y)\right) d y d t \\
& -\int_{\Omega} x^{0}(y) p(T, y) d y d t+\frac{1}{2} \int_{0}^{T} \int_{\Omega}|q(t, y)+v(t, y)|^{2} d y d t \\
& -\frac{1}{2} \int_{0}^{T} \int_{\Omega}|p(T-t, y)|^{2} d y d t+\int_{\Sigma} u(t, y)\langle q(t, y), \nu(y)\rangle d y d t
\end{aligned}
$$

where $u \in H^{2}(\Sigma)$ is the function occurring in (1), and $\nu$ is the unit outward normal to $\Gamma$. It is clear that, for each fixed $(p, q) \in X^{d}, J_{p}(p, q, \cdot)$ is well defined on $L^{2}\left((0, T) \times \Omega ; \mathbb{R}^{n}\right)$ and finite. We observe that $J_{p}$ is convex and lower semicontinuous in the third variable for a fixed $(p, q) \in X^{d}$ and $J_{p}(p, q, 0)=-J_{D}(p, q)$.

We define a kind of Fenchel-Young transform (see e.g. [3]),

$$
J_{p}^{\#}: X^{d} \times X \rightarrow \mathbb{R}
$$

with respect to a duality pairing for the space $L_{2}=L^{2}\left((0, T) \times \Omega ; \mathbb{R}^{n}\right)$ by

$$
\begin{aligned}
J_{p}^{\#}(p, q, x) & =\sup _{v \in L_{2}}\left\{\int_{0}^{T} \int_{\Omega}\langle\nabla x(t, y), v(t, y)\rangle d y d t+\int_{\Omega} x^{0}(y) p(T, y) d y d t\right. \\
& \left.-\frac{1}{2} \int_{0}^{T} \int_{\Omega}|q(t, y)+v(t, y)|^{2} d y d t\right\}-\int_{\Sigma} u(t, y)\langle q(t, y), \nu(y)\rangle d y d t \\
+ & \frac{1}{2} \int_{0}^{T} \int_{\Omega}|p(T-t, y)|^{2} d y d t-\int_{0}^{T} \int_{\Omega} F^{*}\left(t, y, p_{t}(t, y)+\operatorname{div} q(t, y)\right) d y d t .
\end{aligned}
$$

Using the Fenchel-Young transform in $L_{2}$ of the quadratic functional

$$
v \mapsto \frac{1}{2} \int_{0}^{T} \int_{\Omega}|q(t, y)+v(t, y)|^{2} d y d t
$$

(see [3]) we obtain

$$
\begin{aligned}
J_{p}^{\#}(p, q, x)= & -\int_{0}^{T} \int_{\Omega} F^{*}\left(t, y, p_{t}(T-t, y)+\operatorname{div} q(t, y)\right) d y d t \\
& +\int_{0}^{T} \int_{\Omega} x(t, y) \operatorname{div} q(t, y) d y d t+\int_{0}^{T} \int_{\Omega} x(t, y) p_{t}(T-t, y) d y d t
\end{aligned}
$$




$$
\begin{aligned}
& +\frac{1}{2} \int_{0}^{T} \int_{\Omega}|\nabla x(t, y)|^{2} d y d t+\frac{1}{2} \int_{0}^{T} \int_{\Omega}|p(T-t, y)|^{2} d y d t \\
& -\int_{0}^{T} \int_{\Omega} x_{t}(t, y) p(T-t, y) d y d t-\int_{\Omega} x(T, y) x^{1}(y) d y d t .
\end{aligned}
$$

We now provide two lemmas which will be used in the proof of the Duality Principle.

Lemma 3. For any $x \in X$,

$$
\sup _{q \in X_{2}^{d}} \inf _{p \in X_{1}^{d}} J_{p}^{\#}(p, q, x) \leq J(x) .
$$

Proof. Fix $x \in X$. There exists a pair $\left(p^{x}, q^{x}\right) \in X^{d}$ such that

$$
p^{x}(T-t, y)=x_{t}(t, y) \text { and } \nabla x(t, y)=q^{x}(t, y) .
$$

Therefore, using the Fenchel-Young transform of the quadratic functional

in $L^{2}((0, T) \times \Omega ; \mathbb{R})$, we have

$$
v \mapsto \frac{1}{2} \int_{0}^{T} \int_{\Omega}|v(t, y)|^{2} d y d t
$$

$$
\begin{aligned}
& \inf _{p \in X_{1}^{d}}\left\{-\int_{0}^{T} \int_{\Omega} x_{t}(t, y) p(T-t, y) d y d t+\frac{1}{2} \int_{0}^{T} \int_{\Omega}|p(T-t, y)|^{2} d y d t\right\} \\
& \text { and }
\end{aligned}
$$

$$
\begin{aligned}
& \sup _{q \in X_{2}^{d}}\left\{\int_{0}^{T} \int_{\Omega} x(t, y)\left(p_{t}(T-t, y)+\operatorname{div} q(t, y)\right) d y d t\right. \\
& \left.-\int_{0}^{T} \int_{\Omega} F^{*}\left(t, y, p_{t}(T-t, y)+\operatorname{div} q(t, y)\right) d y d t\right\} \leq \int_{0}^{T} \int_{\Omega} F(t, y, x(t, y)) d y d t .
\end{aligned}
$$

Hence we obtain the assertion of the lemma.

Lemma 4. For any $(p, q) \in X^{d}$,

$$
\inf _{x \in X} J_{p}^{\#}(p, q, x)=J_{D}(p, q) .
$$

Proof. As for any $(p, q) \in X^{d}$ there is $x \in X$ such that $\nabla x(t, y)=q(t, y)$, we have

$$
\int_{0}^{T} \int_{\Omega}\langle\nabla x(t, y), q(t, y)\rangle d y d t-\frac{1}{2} \int_{0}^{T} \int_{\Omega}|\nabla x(t, y)|^{2} d y d t=\frac{1}{2} \int_{0}^{T} \int_{\Omega}|q(t, y)|^{2} d y d t
$$

(Fenchel-Young equality, see [3]). Hence from the definition of $J_{p}^{\#}(p, q, x)$ we get the assertion of the lemma. 


\section{Duality and variational principles}

THEOREM 3.

$$
\inf _{x \in X} J(x) \geq \sup _{q \in X_{2}^{d}} \inf _{p \in X_{1}^{d}} J_{D}(p, q) .
$$

Proof. By Lemmas 3 and 4, since

$$
\inf _{a \in A} \sup _{b \in B} I(a, b) \geq \sup _{b \in B} \inf _{a \in A} I(a, b),
$$

we obtain

$$
\begin{aligned}
\inf _{x \in X} J(x) & \geq \inf _{x \in X} \sup _{q \in X_{2}^{d}} \inf _{p \in X_{1}^{d}} J_{p}^{\#}(p, q, x) \\
& \geq \sup _{q \in X_{2}^{d}} \inf _{p \in X_{1}^{d}} \inf _{x \in X} J_{p}^{\#}(p, q, x)=\sup _{q \in X_{2}^{d}} \inf _{p \in X_{1}^{d}} J_{D}(p, q) .
\end{aligned}
$$

We state the necessary conditions, i.e. conditions which the minimizers of the functional $J$ over the set $X$ should satisfy. We observe that due to Corollary 1 it follows that a minimizing sequence of $J$ over the set $X$ may be assumed to be weakly convergent in $L^{2}\left(0, T ; H^{2}(\Omega)\right)$ and $H^{2}((0, T) \times \Omega)$ and strongly in $L^{2}\left(0, T ; H^{1}(\Omega)\right)$.

Theorem 4. Assume that $\inf _{x \in X} J(x)=J(\bar{x})$ for some $\bar{x} \in H^{2}((0, T) \times$ $\Omega$ ) and that the functional $x \mapsto \int_{0}^{T} \int_{\Omega} F(t, y, x(t, y)) d y d t$ is subdifferentiable at $\bar{x}$ (the subdifferential in the sense of convex analysis is taken in $\left.L^{2}\left(0, T ; L^{2}(\Omega)\right)\right)$. Then there exist $\bar{p} \in X_{1}^{d}$ and $\bar{q} \in X_{2}^{d}$ such that for a.e. $(t, y) \in(0, T) \times \Omega$,

$$
\begin{aligned}
& \bar{p}(T-t, y)=\bar{x}_{t}(t, y), \\
& \bar{q}(t, y)=\nabla \bar{x}(t, y), \\
& -\bar{p}_{t}(T-t, y)-\operatorname{div} \bar{q}(t, y)+F_{x}(t, y, \bar{x}(t, y))=0
\end{aligned}
$$

and $J(\bar{x})=J_{D}(\bar{p}, \bar{q})$.

Proof. Let $\bar{x} \in H^{2}((0, T) \times \Omega)$ be such that $J(\bar{x})=\inf _{x^{j} \in X} J\left(x^{j}\right)$ and let $\left\{\left(p^{j}, q^{j}\right)\right\} \subset X^{d}$ denote the sequences corresponding to $\left\{x^{j}\right\}$ according to the definition of $X^{d}$. We define $-\bar{p}_{t}(T-t, y)=\operatorname{div} \bar{q}(t, y)-F_{x}(t, y, \bar{x}(t, y))$, with $\bar{q}$ given by $\bar{q}(t, y)=\nabla \bar{x}(t, y)$, for a.e. $(t, y) \in(0, T) \times \Omega$. It is clear that $(\bar{p}, \bar{q})$ is the limit of a sequence $\left\{\left(p^{j}, q^{j}\right)\right\} \subset X^{d}$. By the definitions of $J, J_{D}$, relations (23), (24) and the Fenchel-Young inequality it follows that

$$
\begin{aligned}
J(\bar{x})= & \int_{0}^{T} \int_{\Omega}\left(\frac{1}{2}|\nabla \bar{x}(t, y)|^{2}-\frac{1}{2}\left|\bar{x}_{t}(t, y)\right|^{2}+F(t, y, \bar{x}(t, y))\right) d y d t \\
& -\int_{\Omega} \bar{x}(T, y) x^{1}(y) d y d t
\end{aligned}
$$




$$
\begin{aligned}
\leq & -\int_{0}^{T} \int_{\Omega} \bar{x}_{t}(t, y) \bar{p}(T-t, y) d y d t+\frac{1}{2} \int_{0}^{T} \int_{\Omega}|\bar{p}(t, y)|^{2} d y d t \\
& +\int_{0}^{T} \int_{\Omega}\langle\nabla \bar{x}(t, y), \bar{q}(t, y)\rangle d y d t-\frac{1}{2} \int_{0}^{T} \int_{\Omega}|\bar{q}(t, y)|^{2} d y d t \\
& -\int_{\Omega} \bar{x}(T, y) x^{1}(y) d y+\int_{0}^{T} F(t, y, \bar{x}(t, y)) d y d t \\
=- & \int_{0}^{T} \int_{\Omega} F^{*}\left(t, y, \bar{p}_{t}(T-t, y)+\operatorname{div} \bar{q}(t, y)\right) d y d t \\
+ & \int_{\Omega} x^{0}(y) \bar{p}(T, y) d y-\int_{\Sigma} u(t, y)\langle\bar{q}(t, y), \nu(y)\rangle d y d t \\
& -\frac{1}{2} \int_{0}^{T}|\bar{q}(t, y)|^{2} d y d t+\frac{1}{2} \int_{0}^{T}|\bar{p}(T-t, y)|^{2} d y d t \\
= & J_{D}(\bar{p}, \bar{q}) .
\end{aligned}
$$

Therefore $J(\bar{x}) \leq J_{D}(\bar{p}, \bar{q})$. By Remark 1 there are two possible sequences $\left\{\left(p^{j}, q^{j}\right)\right\} \subset X^{d}$ corresponding to the sequence $\left\{x^{j}\right\}$ with $q^{j}=\nabla x^{j}$, namely

$$
q^{j}(t, y)=\nabla x^{j}(t, y), \quad p^{j}(T-t, y)=x_{t}^{j}(t, y)
$$

or

(26) $-p_{t}^{j}(T-t, y)=\operatorname{div} q^{j}(t, y)-F_{x}\left(t, y, x^{j}(t, y)\right), \quad q^{j}(t, y)=\nabla x^{j}(t, y)$.

First we investigate the convergence of both sequences. For the sequence (25) we obviously see, by Corollary 1 , that (possibly up to a subsequence) $x_{t}^{j} \rightarrow \bar{x}_{t}=\bar{p}, \nabla x^{j} \rightarrow \nabla \bar{x}=\bar{q}$. Therefore the system (22), (23), (24) is satisfied.

In the case of the sequence (26) we have similarly $q^{j} \rightarrow \bar{q}=\nabla \bar{x}$ and $\operatorname{div} q^{j} \rightarrow \operatorname{div} \bar{q}=\Delta \bar{x}$ in $L^{2}$, possibly up to a subsequence. Moreover,

$$
-\left(-p_{t}^{j}(T-t, y)-\operatorname{div} q^{j}(t, y)\right)=F_{x}\left(t, y, x^{j}(t, y)\right) .
$$

By $\mathbf{G} 4$ we know that $\left\{F_{x}\left(t, y, x^{j}(t, y)\right)\right\}$ is bounded in $L^{\infty}\left(0, T ; L^{2}(\Omega)\right)$ and so in $L^{2}((0, T) \times \Omega)$. From $(27)$ we infer that the sequence $\left\{p_{t}^{j}+\operatorname{div} q^{j}\right\}$ is bounded in $L^{2}$ and up to a subsequence it is also weakly convergent to some $F_{x}(t, y, \bar{x}(t, y))$. Since $x^{j}$ converges almost everywhere pointwise to $\bar{x}$, it follows that $\left\{p_{t}^{j}+\operatorname{div} q^{j}\right\}$ also converges almost everywhere pointwise to $F_{x}(t, y, \bar{x}(t, y))$. We first investigate the convergence of $\left\{p^{j}\right\}$. By $(27)$ we get

$$
-p_{t}^{j}(T-t, y)=-F_{x}\left(t, y, x^{j}(t, y)\right)+\operatorname{div} q^{j}(t, y)
$$

Hence $\left\{p_{t}^{j}\right\}$ and consequently $\left\{p^{j}\right\}$ are bounded in $L^{2}$. Therefore $\left\{p^{j}\right\}$ is 
weakly $L^{2}$ convergent to $\bar{p}$, possibly up to a subsequence. We assume that the subsequences has been selected and denoted by $j$. By Theorem 3 , we have

$$
J(\bar{x}) \geq \liminf _{j \rightarrow \infty} J_{D}\left(p^{j}, q^{j}\right) .
$$

We observe that in both cases $\liminf _{j \rightarrow \infty} J_{D}\left(p^{j}, q^{j}\right) \geq J_{D}(\bar{p}, \bar{q})$. Indeed, by the properties of the sequence $\left\{q^{j}\right\}$,

$$
\begin{aligned}
\liminf _{j \rightarrow \infty}\left(\frac{1}{2} \int_{0}^{T} \int_{\Omega}\left|p^{j}(T-t, y)\right|^{2} d y d t-\frac{1}{2} \int_{0}^{T} \int_{\Omega}\left|q^{j}(t, y)\right|^{2} d y d t\right) \\
=\liminf _{j \rightarrow \infty} \frac{1}{2} \int_{0}^{T} \int_{\Omega}\left|p^{j}(T-t, y)\right|^{2} d y d t-\lim _{j \rightarrow \infty} \frac{1}{2} \int_{0}^{T} \int_{\Omega}\left|q^{j}(t, y)\right|^{2} d y d t \\
\geq \frac{1}{2} \int_{0}^{T} \int_{\Omega}|\bar{p}(T-t, y)|^{2} d y d t-\frac{1}{2} \int_{0}^{T} \int_{\Omega}|\bar{q}(t, y)|^{2} d y d t .
\end{aligned}
$$

Moreover, since $\left\{p_{t}^{j}+\operatorname{div} q^{j}\right\}$ converges pointwise, we have

$$
\begin{aligned}
\lim _{j \rightarrow \infty}\left(\int _ { 0 } ^ { T } \int _ { \Omega } F ^ { * } \left(t, y, p_{t}^{j}\right.\right. & \left.\left.(T-t, y)+\operatorname{div} q^{j}(t, y)\right)\right) \\
& =\int_{0}^{T} \int_{\Omega} F^{*}\left(t, y, \bar{p}_{t}(T-t, y)+\operatorname{div} \bar{q}(t, y)\right) d y d t .
\end{aligned}
$$

The inequality $(28)$ implies equality, i.e. $J(\bar{x})=J_{D}(\bar{p}, \bar{q})$. Since $J(\bar{x})=$ $J_{D}(\bar{p}, \bar{q})$, we have

$$
\begin{aligned}
\int_{0}^{T} \int_{\Omega} F^{*}\left(t, y, \bar{p}_{t}\right. & (T-t, y)+\operatorname{div} \bar{q}(t, y)) d y d t \\
& +\int_{0}^{T} \int_{\Omega} F(t, y, \bar{x}(t, y)) d y d t \frac{1}{2} \int_{0}^{T} \int_{\Omega}|\bar{q}(t, y)|^{2} d y d t \\
& +\frac{1}{2} \int_{0}^{T} \int_{\Omega}|\nabla \bar{x}(t, y)|^{2} d y d t-\int_{\Omega} \bar{x}(T, y) x^{1}(y) d y \\
= & \frac{1}{2} \int_{0}^{T} \int_{\Omega}|\bar{p}(T-t, y)|^{2} d y d t+\frac{1}{2} \int_{0}^{T} \int_{\Omega}\left|\bar{x}_{t}(t, y)\right|^{2} d y d t \\
& +\int_{\Omega} x^{0}(y) \bar{p}(T, y) d y-\int_{\Sigma} u(t, y)\langle\bar{q}(t, y), \nu(y)\rangle d y d t .
\end{aligned}
$$

The functional $x \mapsto \int_{0}^{T} \int_{\Omega} F(t, y, x(t, y)) d y d t$ is convex in $L^{2}$ at $\bar{x}$ by the 
assumption of the theorem, and therefore by (24),

$$
\begin{array}{r}
\int_{0}^{T} \int_{\Omega} F^{*}\left(t, y, \bar{p}_{t}(T-t, y)+\operatorname{div} \bar{q}(t, y)\right) d y d t+\int_{0}^{T} \int_{\Omega} F(t, y, \bar{x}(t, y)) d y d t \\
=\int_{0}^{T} \int_{\Omega} \bar{x}(t, y)\left(\bar{p}_{t}(T-t, y)+\operatorname{div} \bar{q}(t, y)\right) d y d t ;
\end{array}
$$

similarly we get, by (23), the equality

$$
\frac{1}{2} \int_{0}^{T} \int_{\Omega}|\bar{q}(t, y)|^{2} d y d t+\frac{1}{2} \int_{0}^{T} \int_{\Omega}|\nabla \bar{x}(t, y)|^{2} d y d t=\int_{0}^{T} \int_{\Omega}\langle\nabla \bar{x}(t, y), \bar{q}(t, y)\rangle d y d t .
$$

Hence from (30) we deduce that

$$
\frac{1}{2} \int_{0}^{T} \int_{\Omega}|\bar{p}(T-t, y)|^{2} d y d t+\frac{1}{2} \int_{0}^{T} \int_{\Omega}\left|\bar{x}_{t}(t, y)\right|^{2} d y d t=\int_{0}^{T} \int_{\Omega}\left\langle\bar{x}_{t}(t, y), \bar{p}(T-t, y)\right\rangle .
$$

Hence we obtain (22).

6. The proof of Theorem 2. By definition of $X$ and Lemma 2, we see that the functional $J$ is bounded in $X$. We denote by $\left\{x^{j}\right\}$ a minimizing sequence for $J$ in $X$. By Corollary 1 this sequence has a subsequence, denoted again by $\left\{x^{j}\right\}$, converging weakly in $H^{2}((0, T) \times \Omega)$ and strongly in $L^{2}\left(0, T ; H^{1}(\Omega)\right)$, hence also strongly in $L^{2}((0, T) \times \Omega)$ to an element $\bar{x} \in H^{2}((0, T) \times \Omega)$. Moreover, $\left\{x^{j}\right\}$ is also convergent almost everywhere and the sequence $\left\{x_{t}^{j}\right\}$ is strongly convergent in $L^{2}((0, T) \times \Omega)$. Hence $\liminf \operatorname{in}_{j \rightarrow \infty} J\left(x^{j}\right) \geq J(\bar{x})$. Thus $\inf _{x \in X} J\left(x^{j}\right)=J(\bar{x})$. By Theorem 4 we know that $\bar{x}$ along with the corresponding $\bar{p}$ and $\bar{q}$ satisfies (22), (23), (24) and thus equation (1) (see the Remark following Theorem 2). Since the RHS of (1) for that $\bar{x}$ belongs to $\mathcal{L}$, from Theorem 1 we infer that $\bar{x} \in U$ and so $\bar{x} \in X$. This finishes the proof of Theorem 2 .

\section{References}

[1] G. Avalos, I. Lasiecka and R. Triggiani, Uniform stability of nonlinear thermoelastic plates with free boundary conditions, in: Optimal Control of Partial Differential Equations (Chemnitz, 1998), Int. Ser. Numer. Math. 133, Birkhäuser, Basel, 1999, $13-32$.

[2] V. Barbu, I. Lasiecka and M. A. Ramaha, On nonlinear wave equations with degenerate damping and source terms, Trans. Amer. Math. Soc. 357 (2005), 2571-2611.

[3] I. Ekeland and R. Temam, Convex Analysis and Variational Problems, Stud. Math. Appl. 1, North-Holland, 1976.

[4] R. T. Glassey, Blow-up theorems for nonlinear wave equations, Math. Z. 132 (1973), $183-203$. 
[5] H. A. Levine, Instability and nonexistence of global solutions of nonlinear wave equations of the form $P u_{t t}=A u+F(u)$, Trans. Amer. Math. Soc. 192 (1974), $1-21$.

[6] I. Lasiecka, J.-L. Lions and R. Triggiani, Nonhomogeneous boundary value problems for second order hyperbolic operators, J. Math. Pures Appl. 65 (1986), 149-192.

[7] X. Li and J. Yong, Optimal Control Theory for Infinite Dimensional Systems, Birkhäuser, Boston, 1994.

[8] B. S. Mordukhovich and J. P. Raymond, Dirichlet boundary control of hyperbolic equations in the presence of state constraints, Appl. Math. Optim. 49 (2004), 145157.

[9] I. Nowakowska and A. Nowakowski, Dirichlet problem for semilinear hyperbolic equation, Nonlinear Anal. 63 (2005), e43-e52.

[10] L. E. Payne and D. Sattinger, Saddle points and instability of nonlinear hyperbolic equations, Israel J. Math. 22 (1981), 273-303.

[11] B. Yordanov and Q. S. Zhang, Finite-time blowup for wave equations with a potential, SIAM J. Math. Anal. 36 (2005), 1426-1433.

Faculty of Mathematics

University of Łódź

Banacha 22

90-238 Łódź, Poland

E-mail: annowako@math.uni.lodz.pl

Received on 10.12.200\%;

revised version on 1.4.2008 
